\title{
Evaluating Gamut Coverage Metrics for ICC Color Profiles
}

\author{
Radu Velea ${ }^{1}$, Noel Gordon ${ }^{2}$ \\ ${ }^{1}$ Technical Military Academy of Bucharest, Romania \\ ${ }^{2}$ Google Inc
}

\begin{abstract}
The color handling characteristics of computer monitors and other display devices can significantly alter the way an image is perceived by the human eye. Modern cameras and software applications can create vibrant color scenes, and can specify the color range (or gamut) of the scene with an embedded ICC profile [1]. During presentation, differences in the color gamut of the image and the display device require color management to help preserve the color fidelity of the image when shown on different displays. Measurements of the color characteristics of display devices is thus a topic of interest. This paper evaluates the ability of gamut measures, including those commonly used in industry, to classify display gamuts using a user-contributed dataset of ICC display color profiles, and recommends the use of relative metrics for gamut comparison tasks.
\end{abstract}

\section{Introduction}

Colour management - Color gamut is defined as the range of colors a device can reproduce. Color management refers to the translation of color data from one color profile (a gamut specification) to another. In digital imaging systems, the display device, and the image content to be displayed, often have different color gamuts. The underlying color management module (CMM) is responsible for performing color transformations to ensure the user sees a color-correct representation of the image on their display.

The International Color Consortium (ICC) [1] standards define the representation and use of color profiles. In version 2 of the standards, a display profile can be represented with a set of color primaries (colors that define the color spectrum of the profile, commonly given in red, green, and blue components), along with gamma curves and a reference white point. A color transform defines a conversion between an input color profile (usually embedded in the media object) and an output profile (usually associated with a display or operating system setting). The color transform process is mathematical in nature and first requires a

conversion from input to profile connection space (PCS), and then to the output space, with gamma and inverse- gamma operations between (Fig. 1). The resulting transform is then applied to each pixel of the input media. The objective is to maintain color accuracy across heterogeneous displays.

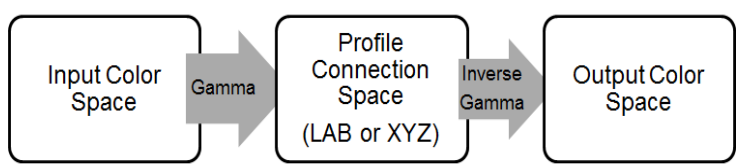

Figure 1. ICC v2 color profile transform.

However, since gamut is a physical device property, if an input color can not be physically reproduced by the destination gamut, unavoidable color distortion results [2], which may be perceived by users. It is therefore important to know (a priori, if possible) the end-user viewing conditions in which the input media will be displayed.

Motivation- Gamut classification of display profiles can yield valuable information on the way color is perceived in end-user systems. Applications that run in multiple-display environments find it useful to detect the capabilities of each display, and choose an appropriate color transform to deliver color-correct content to each display device.

Internet browsers, in particular, run on multiple platforms ranging from mobile, desktop, and highend devices. Browsers have to ensure images on the Internet are displayed in accurate colors, or users will experience different results otherwise (see different colors). A gamut coverage metric can be useful for comparing color profiles, and help front-end applications, such as browsers, make better decisions on how to represent color in end-user systems, and in similar applications, such as the display of medical images, where color is important.

\section{Related Work}

In 1996, HP and Microsoft created sRGB as the standard color space for use on the Internet [3]. For images that do not have an embedded ICC profile, sRGB is used by default. It is reasonable to assume that most content on the Internet today uses sRGB as the color profile [4]. Modern computer monitors have gamuts that are wider than sRGB, and metrics are needed to allow content providers to adjust content gamut for these wider-gamut displays. 
Factors to consider when creating metrics of color gamut include the color space, and a reference profile used for gamut comparison. Note that color gamuts are volumes, and volume computations are slow and complex: fast metrics are required for realtime use.

Profile RGB primaries can be represented in various color spaces: CIE 1931 and CIE 1976 are popular options. Ignoring luminance (brightness), a set of three chromaticity coordinates result, defining a triangular shape when plotted in a $2 \mathrm{D}$ plane. The triangle can be used to compute the gamut area's coverage of the spectrum locus (the range of the colors perceivable by the human eye) in that color space [5].

[6] proposes a method to compute the gamut area in CIE 1976 u'v' chromaticity coordinates, which measures the gamut triangle area, and then divides it by the area of the spectrum locus, from $380 \mathrm{~nm}$ to $700 \mathrm{~nm}$, evaluated at $1 \mathrm{~nm}$ intervals, which is 0.1952 . Multiplying by 100 produces the gamut area coverage as a percentage of the spectrum locus area (called the normalized gamut area, or NGA). The metric is then expanded to compare the profile's gamut with a known reference profile (sRGB, for example). To do this, the authors compute the area of the two gamut triangles, and the area of their intersection, and define a single-number summary metric [6] for comparing color gamuts:

$$
H=a A / A_{0}^{2}
$$

where $\mathrm{a}$ is the area of intersection, $\mathrm{A}$ is the profile gamut area, and $\mathrm{A} 0$ is the reference profile gamut area.

But how reliable is this metric in assessing noninclusive profiles? Assume a reference profile with A_0=50 (its gamut covers half the spectrum locus), and apply the formula given two profiles with A_1 $=70$, a_1 $=50$, A $\_2=80$, a_2 $=35$. The first profile encompasses the reference but has a smaller gamut than the second profile, which in turn overlaps only a portion of the reference. The resulting metrics would be $\mathrm{H} \_1=1.4$ andH_2=1.12. This suggests the first color profile is significantly larger than the second, which is in fact a misstatement.

The choice of CIE $1976 \mathrm{u}^{\prime} \mathrm{v}^{\prime}$ is also problematic. In [7] the authors discuss the choice of CIE $1976 \mathrm{u}^{\prime} \mathrm{v}^{\prime}$ and CIE 1931 xy when computing profile gamut area coverage. They measured the gamut area of the Adobe RGB profile (a de-facto standard in professional color processing) [8], and the DCI-P3 profile (used in digital cinema projection systems) [9]. These profiles have similar characteristics (the DCI-P3 spectrum is slightly shifted towards red [10]). However, computing area-coverage ratios in the reference color spaces resulted in different values: the xy metric produced similar values for gamut area, whereas, u'v' yielded a difference of about $7 \%$ in favor of DCI-P3. By comparing volumecoverage metrics in other color-appearance models, xy was shown to be a much better predictor of gamut volume than u'v' for wide-gamut profiles. Additional data on the inconsistencies between $x y$ and $u^{\prime} v^{\prime}$ metrics are given in the results section.

Considering just xy metrics, [12] proposes a relative gamut representation using NTSC (1953) as the reference. The gamut area of the profile is calculated in xy coordinates, and divided by the NTSC (1953) gamut area (which is 0.1582 if standard Illuminant $\mathrm{C}$ is assumed, or 0.153281 if ICC D50 is used). The metric is called the chromaticity area ratio (CAR):

$$
C A R=A_{w} / A_{r}
$$

where $A_{w}$ is the area of the profile gamut, and $A_{r}$ is the gamut area of the NSTC (1953) reference display.

Another interesting metric is the discernible color number ratio (DCNR) [13]. DCNR aims to provide better accuracy for display gamut comparison by taking into account the number of perceptually discernible object colors a monitor produces. Methods for counting the number of discernible colors within a gamut area are, however, computationally complex, and very sensitive to illumination levels, tolerance factors, and the color difference model used: CIE76, CIE94, CIEDE2000.

In the following sections therefore, we experiment with the lower-complexity metrics (1) and (2) to assess their ability to classify color gamuts using the data directly available from an ICC profile in our measurements.

\section{Implementation}

The main data input will be the ICC profile associated with each user display. The metrics will be built based on the color primaries in the profile, per version 2 of the specification. The primaries are extracted from the profile connection space PCS in XYZ coordinates with D50 white point [1].

The next step converts the XYZ coordinates of the profile primaries to $x y$ and $u^{\prime} v^{\prime}$ color space. The respective equations for these conversions are:

$$
\begin{array}{cc}
x=\frac{X}{X+Y+Z}, \quad y=\frac{Y}{X+Y+Z} \\
u^{\prime}=\frac{4 X}{X+15 Y+3 Z}, \quad v^{\prime}=\frac{9 Y}{X+15 Y+3 Z}
\end{array}
$$


The area for each of the resulting triangles is then calculated. When using metrics involving an additional reference profile, the shape corresponding to the geometric area of intersection with the reference profile is computed. The intersection points for each of the 6 triangle edges are determined, and vertices in common are selected. If the number of vertices is greater than 2, they are sorted counterclockwise, and the enclosed area is computed with the shoelace formula due to Gauss (also known as the Surveyor's formula):

$$
\text { Area }=\frac{1}{2} \sum_{i=1}^{n}\left(x_{i} y_{i \oplus 1}-x_{i \oplus 1} y_{i}\right)
$$

The QCMS (Quick Color Management System) library was used to parse the ICC display profiles. QCMS is a fast and lightweight color management library used by the Firefox and Chrome web browsers to handle color profiles in JPEG, PNG, and WebP images. QCMS provides API to read and parse ICC v2 and ICC v4 profiles, and to perform color transforms on the CPU using SIMD extensions. We expanded the QCMS API to calculate the gamut metrics from equations (1) and (2) in both the CIE 1931 XYZ and CIELUV color spaces, the goal being to determine how many web users have wide-gamut displays, and to allow Chrome developers to use the metric logic in new browser features.

Some of our contributions were included in Skia, which is a 2D graphics engine used by Chrome and Firefox for drawing content on the Web. The current work influenced the decisions to expand color management support in Skia, and provided an efficient implementation of the Skia color cube filter [14] - a color cube represents RGB colors in 3D space [15], and input colors are projected onto this structure (in a process similar to trilinear or tetrahedral interpolation) to produce accurate color space conversions (color transforms). Skia also contains GPU shaders used to apply graphical transformations to input media on the GPU. In future, it is expected that the significant part of the computations for color management will take place on the GPU using shaders.

\section{Results}

\subsection{Single-Number Metrics}

Public databases were used to gather user screen color profiles [16-17] and ICC Taxi color profile database. The input dataset contains about 1300 distinct profiles. The profiles were collected from various user devices, such as desktops, laptops, mobile phones, and other small-form factor screens.
For the single-number summary metric (1) sRGB, which is considered narrow-gamut [18], was used as the reference:

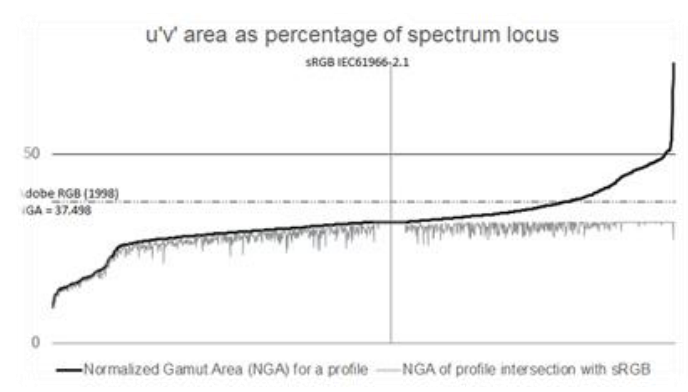

Figure 2. Profile normalized gamut area (NGA [6]) in u'v' sorted left-to- right in ascending order. The sRGB profile is in the middle of the chart. The dashed line is the Adobe RGB normalized gamut area.

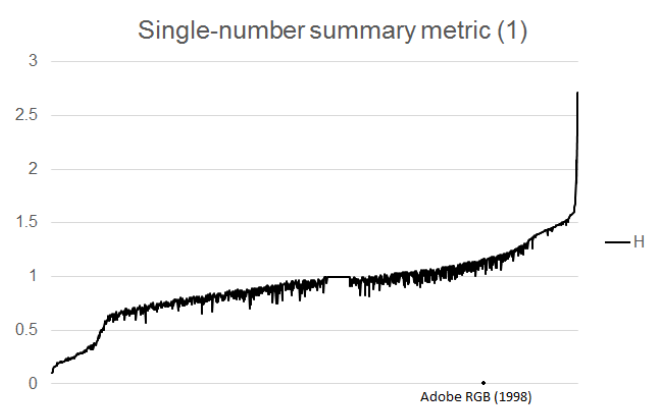

Figure 3. Profile dataset metric $\mathrm{H}$ computed from equation (1).

Figs. 2 and 3, visually demonstrate the effects of including area intersection in a profile gamut metric. The more disjoint two profiles are, the more the metric loses its meaning. If the selected reference profile is either too narrow, or too wide, it would cancel out its effects, and create a chart similar to the NGA graph. Looking at just the NGA graph, we notice that we are dealing with a continuous interval of values. Sorting the dataset in u'v' results in closely packed values. The top $1 \%$ of profiles were originally designed for uses other than computer displays, e.g., the ProPhoto RGB profile used for high-fidelity photographic work. The gamut of ProPhoto RGB, and similar profiles, defines colors that cannot be perceived by the human eye (imaginary colors). As a result, they should be considered an extreme case, and are excluded from our discussions about user display viewing conditions. It would also be unrealistic in the current context to assume a single threshold where a color gamut could be classified as either narrow or wide.

The next set of measurements used CAR from equation (2) and the NTSC (1953) reference assuming Illuminant $\mathrm{C}$ : 


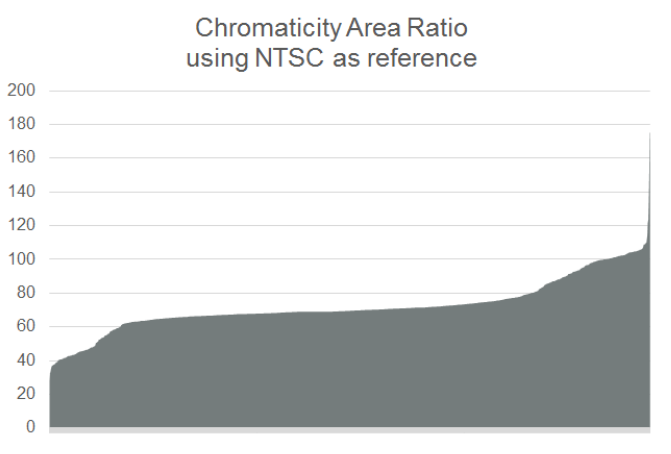

Figure 4. The CAR metric (2) applied to the dataset with NTSC (1953) as the reference and standard Illuminant C [20]. The vertical axis is the metric value expressed as a percentage.

Referring to Fig. 4, most users in this data have sRGB-like conditions. More generally, viewing gamuts form a continuous (rather than discrete) range. Though the xy and u'v' graphs (see Fig. 3) seem similar, there are some important differences. For example, in xy space, the Adobe RGB and DCIP3 profiles are approximately equal $(92.714 \%$ and $92.139 \%$ of NTSC area, respectively), whereas, in the u'v' color space, the DCI-P3 area is about $10 \%$ wider. Similar figures can be found in [19], and recommends the use of xy space coordinates when comparing wide-gamut color profiles.

\subsection{Gamut Coverage as Scalar Triple Product}

ICC standard color profiles define a set of color primaries for red, green, and blue. Each of these primaries is represented in $\mathrm{XYZ}$ as discussed earlier in this paper. The primaries can be thought of as vectors in the 3-dimensional XYZ space. We can therefore compute a geometric score for a color profile gamut by arranging its XYZ primary coordinates in a $3 \times 3$ matrix and calculating the matrix determinant. The resulting value is the scalar triple product (STP), which is the geometric volume of the parallelepiped defined by the primary (red, green, blue) XYZ vectors:

$$
V=R_{X Y Z} \cdot G_{X Y Z} \times B_{X Y Z}
$$

The scalar triple product $\mathrm{V}$ is invariant under circular shifts of its operands, meaning the metric is unchanged if we use any permutation of the color primary vectors. Invalid profiles have a noninvertible matrix (the matrix determinant is not defined) and were excluded from our results.

STP can be used to compare gamut coverage of ICC color profiles in the spectral locus defined by XYZ color space, and plotting the metric computed from the display profile dataset reveals a good correlation with CAR:

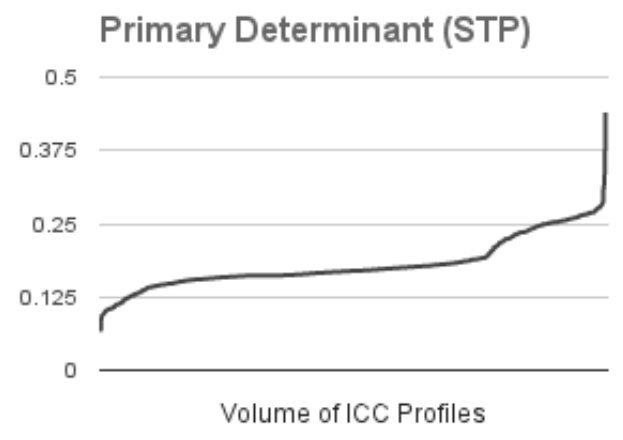

Figure 5. Profile dataset order based on the STP volumetric (6).

The computational cost of the STP metric is very similar to CAR, and the close resemblance suggests it might be a useful measure of gamut area coverage. However, whereas CAR is based on colorimetric principles affecting color profiles, STP is a mathematical by-product of computing a matrix determinant from the XYZ primaries. By superimposing CAR over the STP volume metric, some noise and discrepancy becomes apparent (Fig. $6)$.

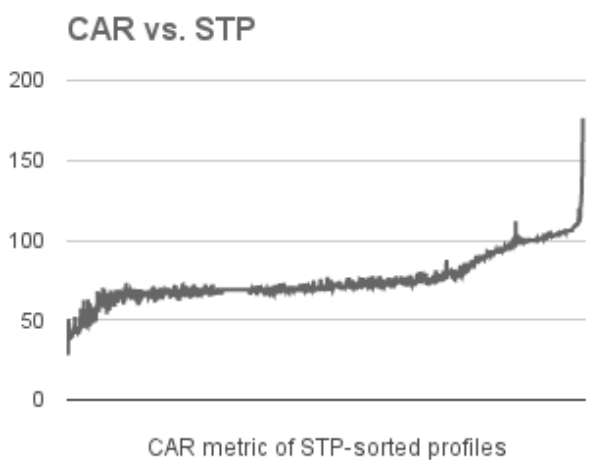

Figure 6. Comparison of CAR and STP metrics.

STP measurement of the ICC sRGB V4 Preference display profile (available from the ICC web-site) produced a score of $18.2 \%$ (of the NTSC 1953 STP at ICC D50). The CAR score of this profile is above $90 \%$, and is correct, based on visual inspection. This observation suggests that STP can misclassify some profiles.

\section{Conclusion}

Gamut classification of displays is useful for detecting how color is perceived in end-user systems. The paper examined the ability of gamut metrics to classify displays using a dataset of end-user display color profiles. 
Gamuts form a continuous range in this user dataset, which suggests that it is not possible to define a numerical threshold to classify display gamuts as either narrow or wide. However, reliable metrics of relative gamut size can be provided using a wide-gamut reference such as NTSC (1953) in xy space.

As a result of this work, the CAR metric has been added to the Chrome browser. Given this display metric, web content providers could adjust the gamut of delivered media to ensure end-users see highfidelity, color-correct representations of the media on their displays.

\section{References}

[1] International Color Consortium, "Introduction to the ICC profile format." http://www.color.org/iccprofile.xalter, 2016. Access Date: 12 May, 2016.

[2] Cambridge in Colour, "Color Management: Color Space Conversion," http://www.cambridgeincolour.com /tutorials/color-space-conversion.htm. Access Date: 12 March, 2017.

[3] Michael Stokes, Srinivasan Chandrasekar, Matthew Anderson, Ricardo Motta, "A Standard Default Color Space for the Internet - sRGB,"https://www.w3.org/ Graphics/Color/sRGB.html, 1996. Access Date: 12 May, 2016.

[4] R. Rehák, P. Bodrogi, J. Schanda, "On the use of the sRGB colour space," pp. 165-170, Displays Volume 20, Issue 4, Elsevier, 1999.

[5] Francisco Miguel Martinez Verdu, Maria Jose Luque Cobija, Pascual Capilla Perea, Jaume Pujol Ramo, "Concerning the calculation of the color gamut in a digital camera,” pp. 399-410, John Wiley \& Sons Ltd, 2006.

[6] Society for Information Display, Information Display Measurements Standard. International Committee for Display Metrology, 2012.

[7] Kenichiro Masaoka, Yukihiro Nishida, "Metric of color-space coverage for wide-gamut displays," pp. 78027808, Optics Express, Vol. 23, Issue 6, 2015.

[8] Adobe Systems Inc., “Adobe RGB (1998) Color Image Encoding," 2005.

[9] Society of Motion Picture and Television Engineers, SMPTE RP 431-2:2011, "D-Cinema Quality - Reference Projector and Environment," 2011.

[10] Jeff Yurek, "How much color gamut do displays really need? Part 3: Existing color gamut standards." http://dotcolor.com/category/color-gamut-standards, 2013. Access Date: 12 May, 2016.

[11] G. Wyszecki and W. S. Stiles, Color Science. 7th ed. New York: Wiley, 1982.
[12] Senfar Wen, "Representations of relative display gamut size," pp. 18-23, Journal of Display Technology (Volume:4, Issue:1), IEEE, 2008.

[13] Senfar Wen, "Display gamut comparison with number of discernible colors," pp. 043001-1 - 043001-8, Journal of Electronic Imaging, Volume 15, Issue 4, 2006.

[14] Skia Graphics Library, "Skia's New Approach to SIMD" https://skia.org/dev/contrib/simd, 2017. Access Date: 28 January, 2017.

[15] Jose-Luis Lisani, Antoni Buades, Jean-Michel Morel, "Image Color Cube Dimensional Filtering and Visualization", Image Processing on Line, June, 2011.

[16] Digitalversus, "Colour Calibration Profiles for your Monitor," http://www.digitalversus.com/lcd-monitor/ colour-calibration-profiles-your-monitor-a424.html". Access Date: 12 May, 2016.

[17] TFT Central, "ICC profiles and monitor settings database." http://www.tftcentral.co.uk/articles/icc_ profiles.htm, Aug 2016. Access Date: 12 May, 2016.

[18] Ying Xiong, Kate Saenko, Trevor Darrell, Todd Zickler, "From pixels to physics: Probabilistic color derendering," pp. 358-365, IEEE Conference on Computer Vision and Pattern Recognition (CVPR), 2012.

[19] Kid Jansen, “The Pointer's Gamut - The coverage of real surface colors by RGB color spaces and wide gamut displays."

http://www.tftcentral.co.uk/articles/pointers_gamut.htm, 2014. Access Date: 12 March, 2017.

[20] ASTM E.308 - 01, "Standard Practice for Computing the Color of Objects Using the CIE System," 2001. 